\title{
Radiation From a Vertical Antenna Over a Curved Stratified Ground
}

\author{
James R. Wait
}

\begin{abstract}
The problem of a radial electric dipole outside a concentrically stratified spherical conductor, such as the earth, is formulated. The solution is facilitated by considering the analogous nonuniform transmission line for the radial modes. The general result is then transformed to a Watson-type residue or azimuthal mode series, which reduces to the well-known result for the homogeneous earth as a special case. Following a method introduced recently by Bremmer, the residue series is converted to an alternative expansion, which is more suitable at short distances. The leading term of this new expansion corresponds to the case of the transmitter and receiver over a plane stratified conducting earth.
\end{abstract}

\section{Introduction}

In an earlier paper by the author [1] ${ }^{1}$ expressions were derived for the fields of a vertical electric dipole over a plane stratified ground. In a further paper [2], the solution was extended to arbitrary antenna heights and numerical values of the attenuation factor were given. In the latter paper it was mentioned that the effect of the earth's curvature could be accounted for. It is the purpose of this paper to develop the theory for propagation over a curved earth with concentric stratifications.

The method of solution is a direct extension of Watson's [3] method to obtain a solution for the electric dipole radiating over a homogeneous sphere. It therefore does not seem necessary to outline the steps in the derivation in detail. A particular novel feature of the formulation, however, is that the boundary conditions at the surface of the sphere are specified by a surface impedance. The final expressions for the fields are then also applicable to propagation over other modified surfaces, such as a sphere with azimuthal periodic corrugations. Using a method suggested recently by $H$. Bremmer, ${ }^{2}$ an expression for the field is then obtained that is very suitable for computation when the receiver is near the optical horizon. Finally, some numerical results are presented for various frequencies employing typical values of the ground constants over both a homogeneous and stratified earth.

\section{Formal Solution}

The source of the field is considered to be an electric current element $I d s$ oriented in the radial direction to the spherical earth of radius $a_{1}$. Choosing a spherical coordinate system $(r, \theta, \phi)$, the surface of the earth is then defined by $r=a_{1}$, and the dipole or current element is located at $r=b$ and $\theta=0$. The fields can now be expressed in terms of a Hertz

\footnotetext{
${ }_{2}^{1}$ Figures in brackets indicate the literature references at the end of this paper
}

vector, which has only a radial component $U$, as follows:

$$
\left.\begin{array}{l}
E_{r}=k^{2}+\frac{\partial^{2}}{\partial r^{2}}(r U) \\
E_{\theta}=\frac{1}{r} \frac{\partial^{2}}{\partial r \partial \theta}(r U) \\
H_{\phi}=-i \epsilon \omega \frac{\partial U}{\partial \theta}
\end{array}\right\} \text { for } r>a_{1}
$$

where $k=2 \pi /$ free-space wavelength, and $\epsilon$ is the dielectric constant of free space, $\left(=8.854 \times 10^{-12}\right)$, in mks units. A time factor $\exp (i \omega t)$ has been implied. The function $U$ satisfies the equation

$$
\left(\nabla^{2}+k^{2}\right) U=C \frac{\delta(r-b) \delta(\theta)}{2 \pi r^{2} \sin \theta},
$$

where the $\delta$ 's are the Dirac delta or impulse function. The factor $2 \pi r^{2} \sin \theta$ is the Jacobian of the transformation from rectangular to spherical coordinates. The constant is chosen so that $U$ has the proper singularity at the dipole, that is

$$
U \rightarrow \frac{e^{-i k R}}{4 \pi i \omega \epsilon R} I d s \text { for } R \rightarrow 0,
$$

where $R=\left[r^{2}+b^{2}-2 b r \cos \theta\right]^{1 / 2}, \epsilon=8.854 \times 10^{-12}$, and therefore $C=(i / \omega \epsilon) I d s$.

The field $U$ is now written as the sum of two parts $U_{e}+U_{s}$, where $U_{e}$ has the proper dipole singularity at $R=0$, and $U_{s}^{e}$ is finite at that point. As $U_{8}$ is a solution of the homogeneous wave equation, it can be written in the form

$$
U_{s}=\frac{i k C}{4 \pi} \sum_{n=0}^{\infty}(2 n+1) A_{n} h_{n}^{(2)}(k r) P_{n}(\cos \theta),
$$

where $h_{n}^{(2)}(\mathrm{kr})$ is the spherical Hankel function of the second kind, which assures outgoing waves at infinity, and $P_{n}(\cos \theta)$ is the Legendre function. The corresponding representation for $U_{e}$ is [4] 


$$
\begin{aligned}
U_{e} & =\frac{i k C}{8 \pi} \sum_{n=0}^{\infty}(2 n+1) h_{n}^{(1)}(k r) h_{n}^{(2)}(k b) P_{n}(\cos \theta) \text { for } r<b \\
& =\frac{i k C}{8 \pi} \sum_{n=0}^{\infty}(2 n+1) h_{n}^{(2)}(k r) h_{n}^{(1)}(k b) P_{n}(\cos \theta) \text { for } r>b
\end{aligned}
$$

The coefficients $A_{n}$ are now found from the boundary condition, that

$$
E_{\theta}=-Z H_{\phi} \text { at } r=a_{1},
$$

which can be rewritten

$$
\left[\frac{1}{r} \frac{\partial}{\partial r} r U=Z i \epsilon \omega U\right]_{r=a_{1}} .
$$

In other words, it is assumed that the surface of the earth exhibits the property of surface impedance. $Z$ is taken to be equal to the ratio of the tangential electric and magnetic fields for a vertically polarized plane wave at grazing incidence on a plane stratified earth. This step in the analysis leads to a great simplification, and it is justified in the appendix. It then readily follows that

$A_{n}=-\frac{h_{n}^{(1)}\left(k a_{1}\right)}{h_{n}^{(2)}\left(k a_{1}\right)}\left[\frac{\frac{1}{x} \frac{d}{d x} \log x h_{n}^{(1)}(x)-\frac{i \Delta}{x}}{\frac{1}{x} \frac{d}{d x} \log x h_{n}^{(2)}(x)-\frac{i \Delta}{x}}\right]_{x=k a_{1}} h_{n}^{(2)}(k b)$,

where $\Delta=\epsilon \omega Z / k=Z / 120 \pi$ and where $h_{n}^{(1)}(x)$ is the spherical Hankel function of the first kind. The total field is then of the form

$$
U=\sum_{n=0}^{\infty}(2 n+1) f(n) P_{n}(\cos \theta) .
$$

Following the process developed by Watson [3], the summation is transformed into the following contour integral:

$$
U=i \int_{c_{1}+c_{2}} \frac{n d n}{\cos n \pi} f\left(n-\frac{1}{2}\right) P_{n}-1 / 2[\cos (\pi-\theta)],
$$

where the contour $c_{1}+c_{2}$ encloses the positive real axis, as illustrated in figure 1 . Noting that the poles of the integrand are located at $n=1 / 2,3 / 2,5 / 2$, ...., etc., it can be readily verified by the theorem of residues that this integral is equivalent to eq (9). Now, since $f(n-1 / 2)$ is an even function of $n$, the part of the contour $c_{1}$ above the real axis can be replaced by the contour $c_{1}^{\prime}$, which is located just below the negative real axis. The contour $c_{1}^{\prime}+c_{2}$ is now entirely equivalent to $L$, a straight line running along just below the real axis. Replacing $n-1 / 2$ by $\nu$, the contour representation for $U$ takes the form

$$
U=-i \int_{L} \frac{(\nu+1 / 2)}{\sin \nu \pi} f(\nu) P_{\nu}[\cos (\pi-\theta)] d \nu .
$$

It is to be noted that this manipulation of the contours is simplified because $f(n-1 / 2)$ is an even

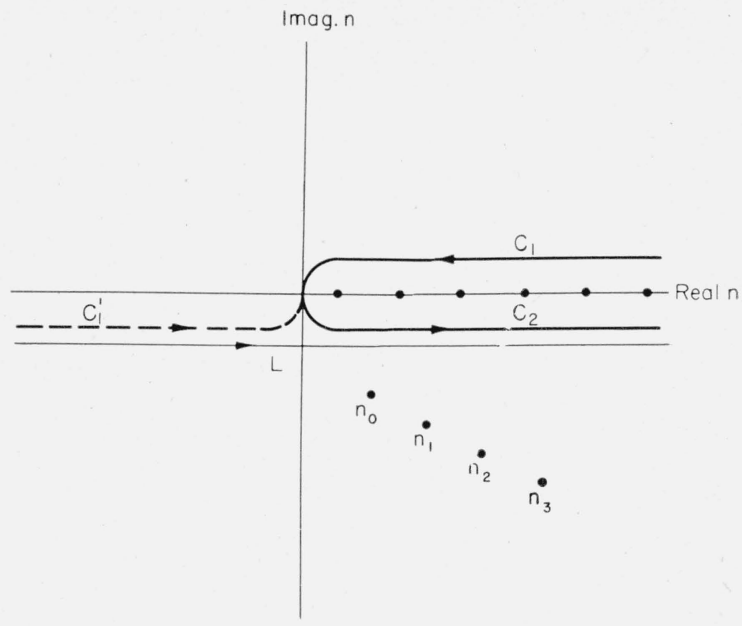

Figure 1. Complex $n$ plane showing the contours and the zeros of $\cos n \pi$ and zeros of the denominator of equation (8).

function of $n$ as a consequence of the method of formulation. In the usual treatment for the homogeneous sphere, $f(n-1 / 2)$ is not an even function of $n$, and the deformation of the contour becomes more intricate.

The next step in the analysis is to close $L$ by an infinite semicircle in the negative half-plane. The contribution from this portion of the contour vanishes as the radius of the semicircle approaches infinity. The reasoning for this fact follows directly from Watson's argument for the homogeneous sphere. The value of the integral for $U$ along the contour $L$ is now equal to the sum of the residues of the integrand evaluated at the poles $\nu_{s}$ of $f(\nu)$ located in the lower half-plane. It then follows that $U$ is proportional to

$$
\sum_{s=0}^{\infty} \frac{\left(\nu_{s}+1 / 2\right) h_{\nu_{s}}^{(2)}(k b) h_{\nu_{s}}^{(2)}(k r) P_{\nu_{s}}[\cos (\pi-\theta)]}{\sin \left(\pi \nu_{s}\right)\left[\frac{\partial M(\nu)}{\partial \nu}\right]_{\nu=\nu_{s}}\left[h_{\nu_{s}}^{(2)}\left(k a_{1}\right)\right]^{2}}
$$

where the function $M(\nu)$ is defined by

$$
M(\nu)=\left[\frac{1}{x} \frac{d}{d x} \log x h_{n}^{(2)}(x)-i \frac{\Delta}{x}\right]_{x=k a_{1}}
$$

and the poles $\nu_{s}$ are the solutions of $M(\nu)=0$. Again, as a result of the formulation, the equation here for the determination of the roots is relatively simple. Making the usual approximation [1] that $h_{n}^{(2)}(x)$ can be represented by Hankel functions of order $1 / 3$, eq (13) can be replaced by

$$
\delta e^{i \pi / 3} \frac{H_{2 / 3}^{(2)}\left[(1 / 3)\left(-2 \tau_{s}\right)^{3 / 2}\right]}{H_{1 / 3}^{(2)}\left[(1 / 3)\left(-2 \tau_{s}\right)^{3 / 2}\right]}=-\left(-2 \tau_{s}\right)^{-1 / 2},
$$

where

$$
\delta=-i \frac{120 \pi}{\left(k a_{1}\right)^{1 / 3} Z} \text { and } \tau_{s}=\frac{\nu_{s}-k a_{1}}{\left(k a_{1}\right)^{1 / 3}} .
$$


For a homogeneous earth whose propagation constant is $\gamma_{1}$, it follows that

$$
Z=120 \pi \frac{\gamma_{0}}{\gamma_{1}}\left[1-\frac{\gamma_{0}^{2}}{\gamma_{1}^{2}}\right]^{1 / 2},
$$

with $\gamma_{0}=i k$, and therefore

$$
\delta=-\frac{i}{\left(k a_{1}\right)^{1 / 3}} \frac{\gamma_{1}^{2} / \gamma_{0}^{2}}{\left[\left(\gamma_{1}^{2} / \gamma_{0}^{2}\right)-1\right]^{1 / 2}},
$$

which is identical to the value given by Bremmer [4]. For a two-layer ground whose upper stratum of thickness $l$ has propagation constant $\gamma_{1}$, the surface impedance $Z$ is given by (see appendix)

$$
Z=120 \pi \frac{\gamma_{0}}{\gamma_{1}}\left[1-\frac{\gamma_{0}^{2}}{\gamma_{1}^{2}}\right]^{1 / 2} Q
$$

where

$$
Q=\tanh \left[P+\left(\gamma_{1}^{2}-\gamma_{0}^{2}\right)^{1 / 2} l\right]
$$

and

$$
P=\tanh ^{-1}\left[\frac{\gamma_{1}^{2}}{\gamma_{2}^{2}}\left(\frac{\gamma_{2}^{2}-\gamma_{0}^{2}}{\gamma_{1}^{2}-\gamma_{0}^{2}}\right)^{1 / 2}\right]
$$

with $\gamma_{2}$ as the propagation constant of the lower medium. The correction factor $Q$, which approaches unity for $l \rightarrow \infty$, can be evaluated with the aid of charts of hyperbolic functions of complex argument. The expression for $Q$ for a stratified ground for any number of layers has been given previously along with some numerical values for special cases [5].

Equation (12) for $U$ can now be considerably simplified by replacing the Legendre function by its leading term in its asymptotic expansion and the functions $h_{\nu}^{(2)}(k b)$ and $h_{\nu}^{(2)}(k r)$ by their Hankel approximations to lead to

$$
U=2 U_{0} \sqrt{-2 \pi i X} \sum_{s=0}^{\infty} f_{s}\left(h_{1}\right) f_{s}\left(h_{2}\right) \frac{e^{-i \tau_{s} X}}{2 \tau_{s}-1 / \delta^{2}}
$$

with

$$
f_{s}\left(h_{i}\right)=\left[\frac{X_{i}^{2}-2 \tau_{s}}{-2 \tau_{s}}\right]^{1 / 2} \frac{H_{1 / 3}^{(2)}\left[\frac{1}{3}\left(X_{i}^{2}-2 \tau_{s}\right)^{3 / 2}\right]}{H_{1 / 3}^{(2)}\left[\frac{1}{3}\left(-2 \tau_{s}\right)^{3 / 2}\right]}
$$

and where

$U_{0}=\frac{I d s e^{-i k a_{1} \theta}}{4 \pi i \omega \epsilon a_{1} \theta}, \quad h_{1}=r-a_{1}$,

and

$$
h_{2}=b-a_{1}, \quad X=\left(k a_{1}\right)^{1 / 3} \theta,
$$

$$
X_{i}=\left(k a_{1}\right)^{1 / 3}\left(2 h_{i} / a_{1}\right)^{1 / 2} \text { for } i=1,2 .
$$

The preceding equations are identical in form to that obtained for the homogeneous earth as given by Watson [3], Bremmer [4], Friedman [6], and others. It is important to note that the quantities $\rho, \delta$, and the roots $\tau_{s}$ are dependent on the electric constants and the nature of the stratification of the earth. Bremmer [4] has given very adequate for- mulas for $\tau_{s}$ in terms of powers of $\delta$. These can be used directly for the stratified earth. They can also be used for a sphere with a corrugated surface if the appropriate value of the surface impedance is employed [7].

\section{Modified Flat Earth Formula}

The so-called residue series for $U$ could be used for calculations for propagation over a curved earth. The series, however, converges poorly for distances near the optical horizon. It would be desirable to transform the residue series formula to a new type of expansion where the first term corresponds to the radiation of a dipole over a plane stratified earth. Succeeding terms would then be preferably in proportion to inverse powers of $k a_{1}$. In the limiting case when $k a_{1}$ tends to infinity, the expression $U$ should correspond to the situation treated previously. A method of obtaining expansion formula of this type is mentioned briefly by Bremmer in his book [4]. Very recently he has described to the author an alternative procedure, which he illustrated for the case when the transmitter and receiver are located on the surface of a homogeneous spherical earth. His method will be employed here in the case when the transmitter and receiver are not both on the earth. Furthermore, as will be shown, the method also is applicable to stratified and corrugated surfaces. The first step is to express the field in terms of a contour integral as follows:

$$
\frac{i U(\rho)}{2 \rho^{1 / 2} U_{0}}=\frac{1}{2 \pi i} \int_{c-i \infty}^{c+i \infty} \frac{\pi^{1 / 2} f\left(p, h_{1}\right) f\left(p, h_{2}\right) e^{p_{\rho}} d p}{1+e^{i \pi / 2} p^{1 / 2} A(p)},
$$

where $\rho=i X / 2 \delta^{2}$ and where ${ }^{-} \mid A(p)$ and $f(p, h)$ are chosen as follows:

$$
A(p)=\frac{H_{2 / 3}^{(2)}\left(\frac{p^{3 / 2}}{3 \delta^{3}}\right)}{H_{1 / 3}^{(2)}\left(\frac{p^{3 / 2}}{3 \delta^{3}}\right)}
$$

and

$$
f\left(p, h_{i}\right)=\left[\frac{X_{i}^{2}+\left(p / \delta^{2}\right)}{\left(p / \delta^{2}\right)}\right]^{1 / 2} \frac{H_{1 / 3}^{(2)}\left[\frac{1}{3}\left(X_{i}^{2}+p / \delta^{2}\right)^{3 / 2}\right]}{H_{1 / 3}^{(2)}\left[\frac{1}{3}\left(p / \delta^{2}\right)^{3 / 2}\right.},
$$

for $i=1,2$; and $c$ is some positive constant.

It can be noted that the poles $p_{s}$ of the integrand are determined by the solution of

$$
H_{1 / 3}^{(2)}\left[\frac{p_{s}^{3 / 2}}{3 \delta^{3}}\right]+e^{i \pi / 3} p_{s}^{1 / 2} H_{2 / 3}^{(2)}\left[\frac{p_{s}^{3 / 2}}{3 \delta^{3}}\right]=0 .
$$

If $p_{s}$ is replaced by $2 \delta^{2} \tau_{s} e^{+i \pi}$ this equation is identical to eq (14) for the determination of the roots $\tau_{s}$. It can be readily verified that the sum of the residues evaluated at the poles $p_{s}$ leads back to eq (21).

Recognizing that the right-hand side of eq (23) is in the form of an inverse Laplace Transform, it can 
be written in Heaviside's operational notation [8] as follows:

$$
\frac{i U(\rho) \overline{1}}{2 \rho^{1 / 2} U_{0}}=F(p)
$$

where

$$
F(p)=\frac{\pi^{1 / 2} p f\left(p, h_{1}\right) f\left(p, h_{2}\right)}{1+e^{i \pi / 3} p^{1 / 2} A(p)}
$$

and

$$
\overline{1}=\left\{\begin{array}{l}
1 \text { for } \rho>0 \\
0 \text { for } \rho<0 .
\end{array}\right.
$$

The technique is now to expand $F(p)$ in an asymptotic expansion in powers of $1 / p$ and then to invert each term to obtain series expansion for $\rho$ in powers of $\delta^{3}$. It is convenient for the moment to consider $h_{2}=0$ so that $f\left(p, h_{2}\right)=1$. Each of the Hankel functions is now expanded in an asymptotic expansion, with due regard being paid to the phase of arguments. Note that

$$
+\pi>\frac{3}{2} \arg \cdot p_{s}-3 \text { arg. } \delta-3 \pi>-2 \pi,
$$

which suggests replacing the arguments $z$ by $z e^{-3 i \pi}$ and then employing the relation

$$
H_{\nu}^{(2)}\left(z e^{-3 i \pi}\right)=-\frac{\sin 2 \pi \nu}{\sin \pi \nu} H_{\nu}^{(2)}(z) .
$$

$$
\begin{aligned}
\frac{F(p)}{i \pi^{1 / 2}}=-\frac{i p e^{-\alpha p^{1 / 2}}}{1-i p^{1 / 2}}+\delta^{3} & {\left[\frac{i}{2\left(1-i p^{1 / 2}\right)^{2}}-\frac{\alpha}{2\left(1-i p^{1 / 2}\right)}\right] e^{-\alpha p^{1 / 2}} } \\
& +\delta^{6}\left[\frac{5}{8} \frac{1}{p^{3 / 2}\left(1-i p^{1 / 2}\right)^{2}}-\frac{i}{4 p\left(1-i p^{1 / 2}\right)^{3}}+\frac{\alpha}{4 p\left(1-i p^{1 / 2}\right)^{2}}+\frac{5 i \alpha}{8 p^{3 / 2}\left(1-i p^{1 / 2}\right)}\right] e^{-\alpha p^{1 / 2}}
\end{aligned}
$$

plus terms containing $\delta^{9}, \delta^{12}$, etc.

The final step in the analysis is to find the corresponding functions of $\rho$ for each term on the righthand side of the above equation. The necessary operational pairs can be derived from the basic relation [9],

$$
M(g, \rho) \overline{1}=\frac{e^{-\alpha p 1 / 2}}{1+g p^{1 / 2}}-1
$$

where

$$
M(g, \rho)=\operatorname{erfc}\left(\frac{\alpha}{2 p^{1 / 2}}\right)-e^{\left(\frac{\alpha}{g}+\frac{\rho}{g^{2}}\right)} \operatorname{erfc}\left(\frac{\alpha}{2 \rho^{1 / 2}}+\frac{\rho^{1 / 2}}{g}\right)
$$

with

$$
\operatorname{erfc}(z)=\frac{2}{\pi^{1 / 2}} \int_{z}^{\infty} e^{-x^{2}} d x
$$

On making this conversion, the validity of the asymptotic expansions is established. It then follows that

$$
\begin{gathered}
f\left(p, h_{1}\right)=e^{-\left(z_{1}-z_{2}\right) i}\left[1+\frac{\delta^{2} X_{1}^{2}}{p}\right]^{-1 / 4}\left\{1-i \frac{5}{72}\left[\frac{1}{z_{2}}-\frac{1}{z_{1}}\right]\right. \\
\left.+\frac{335}{10368} \frac{1}{z_{2}^{2}}-\frac{385}{10368} \frac{1}{z_{1}^{2}}+\frac{25}{5184} \frac{1}{z_{1} z_{2}}\right\}
\end{gathered}
$$

plus terms in $\frac{1}{z_{1}^{3}}$, etc., with

and

$$
z_{1}=\frac{1}{3}\left[\frac{p}{\delta^{2}}\right]^{3 / 2}\left[1+\frac{X_{1}^{2} \delta^{2}}{p}\right]^{3 / 2} e^{-3 i \pi}
$$

$$
z_{2}=\frac{1}{3}\left[\frac{p}{\delta^{2}}\right]^{3 / 2} e^{-3 i \pi} .
$$

To further simplify the above expression for $f(p, h)$, the factor $\left(1+X^{2} \delta^{2} / p\right)^{3 / 2}$ is expanded in a binominal series, and terms containing $X_{1}^{4}, X_{1}^{6}$, etc. are neglected. After some algebraic manipulation, it follows that

$$
f\left(p, h_{1}\right) \simeq e^{-\alpha p^{1 / 2}}\left[1-\frac{i \alpha \delta^{3}}{2 p}-\frac{5}{8} \frac{\alpha \delta^{6}}{p^{3 / 2}}\right]
$$

plus terms containing $\alpha^{2} \delta^{6}, \alpha^{3} \delta^{9}$, etc., where $\alpha=k h_{1} \Delta$. When this formula for $f\left(p, h_{1}\right)$ is substituted into eq $(30)$, it is readily shown that
For example,

$$
\frac{e^{-\alpha p^{1 / 2}}}{\left(1-i p^{1 / 2}\right)^{2}}=\left[g \frac{\partial}{\partial g} M(g, \rho)+M(g, \rho) \quad \underset{s=-i}{1}\right.
$$

so that

$$
\begin{aligned}
& \frac{i \pi^{1 / 2} e^{-\alpha p^{1 / 2}}}{\left(1-i p^{1 / 2}\right)^{2}}=\left\{i \pi^{1 / 2} \operatorname{erfc}\left[\frac{\alpha}{2 \rho^{1 / 2}}\right]+2 \rho^{1 / 2} e^{-\alpha^{2 / 4 \rho}}\right. \\
& \left.-i \pi^{1 / 2}(1+2 \rho-i \alpha) e^{-(\rho-i \alpha)} \operatorname{erfc}\left[\frac{\alpha}{2 \rho^{1 / 2}}+i \rho^{1 / 2}\right]\right\} \overline{1}
\end{aligned}
$$

The other pairs can be derived in a similar manner by further differentiations with respect to the param- 
eter $g$ and then setting $g=-i$. The final result after collecting like terms is

$$
\begin{array}{r}
\frac{U(\rho)}{2 U_{0}}=e^{-a^{2} / 4 \rho}-i(\pi \rho)^{1 / 2} e^{-(\rho-i \alpha)} \operatorname{erfc}\left[\frac{\alpha}{2 \rho^{1 / 2}}+i \rho^{1 / 2}\right] \\
+\delta^{3} \rho^{1 / 2}\left[\rho^{1 / 2} e^{-\alpha^{2} / 4 \rho}-\frac{i \pi^{1 / 2}(1+2 \rho)}{2} e^{-(\rho-i \alpha)} \operatorname{erfc}\left(\frac{\alpha}{2 \rho^{1 / 2}}+i \rho^{1 / 2}\right)\right. \\
\left.\left.+\frac{i \pi^{1 / 2}}{2}(1+i \alpha) \operatorname{erfc} \frac{\alpha}{2 \rho^{1 / 2}}\right)\right]
\end{array}
$$

plus terms containing $\delta^{6}, \delta^{9}$, etc.

The coefficient of the $\delta^{6}$ term is not written out here as it is quite cumbersome and in itself would not be suitable for computation. It is constructive at this stage to express $U(\rho)$ in terms of the parameter $w$ defined by

$$
w=\rho\left(1+\frac{h_{1}}{\Delta a_{1} \theta}\right)^{2},
$$

whence

$$
\begin{aligned}
\frac{U(\rho)}{2 U_{0}} \simeq & e^{-i k h_{1}^{2} / 2 a_{1} \theta}\left\{1-i(\pi \rho)^{1 / 2} e^{-v} \operatorname{erfc}\left(i w^{1 / 2}\right)\right. \\
& \left.+\delta^{3}\left[\rho-i(\pi \rho)^{1 / 2} \frac{(1+2 \rho)}{2} e^{-w} \operatorname{erfc}\left(i w^{1 / 2}\right)\right]\right\} \\
& +\delta^{3}\left[\frac{i(\pi \rho)^{1 / 2}}{2}\left(1+i k h_{1} \Delta\right) \operatorname{erfc}\left(\frac{-i k h_{1}^{2}}{2 a_{1} \theta}\right)^{1 / 2}\right]
\end{aligned}
$$

For the homogeneous earth, the factor $\Delta=$ $\left(\gamma_{0}^{2} / \gamma_{1}^{2}\right)\left(\gamma_{1}^{2} / \gamma_{0}^{2}-1\right)^{1 / 2}$, and if $a_{1}$ the radius tends to infinity or $\delta=0$, the remaining first term corresponds to the well-known formula for the attenuation factor of a dipole over a flat earth [10]. $\rho$ is then the numerical distance of Sommerfeld [11]. It is interesting to note that the form of the first term is identical to the result obtained by Hufford [12], using an integral-equation formulation. When the ground is stratified such that $\Delta=\epsilon \omega Z / k$, where $Z$ is the surface impedance, the first term corresponds to the result obtained previously for the dipole over the plane stratified earth $[1,2]$.

It now appears that, for small heights such that $k h_{1}^{2} / 2 a \theta$ is small compared to one, the height gain function is simply $1+i \alpha$, which is a common factor of at least the first three terms in the expansion in powers of $\delta^{3}$. To this approximation the final result is expressed conveniently as

$$
\begin{aligned}
& \frac{U(\rho)}{2 U_{0}}=W(\rho)=G\left\{F_{0}(\rho)-\frac{\delta^{3}}{2}\left[1-i(\pi \rho)^{1 / 2}-(1+2 \rho) F_{0}(\rho)\right]\right. \\
& \left.+\delta^{6}\left[1-i(\pi \rho)^{1 / 2}(1-\rho)-2 \rho+\frac{5}{6} \rho^{2}+\left(\frac{\rho^{2}}{2}-1\right) F_{0}(\rho)\right] \ldots\right\}
\end{aligned}
$$

where

$$
\begin{aligned}
& F_{0}(\rho)=1-i(\pi \rho)^{1 / 2} \operatorname{erfc}\left(i \rho^{1 / 2}\right) \\
& G \simeq(1+i \alpha)(1+i \beta) \text { with } \beta=k h_{2} \Delta .
\end{aligned}
$$

At distances from the antenna greater than a few wavelengths the quantity $U(\rho)$ is proportional to the vertical component of the electric field, and correspondingly $2 U_{0}$ is proportional to the field of an identical source over a flat perfectly conducting earth. The field strength $E$, in millivolts per meter, at a distance $D_{\mathrm{km}}$, in kilometers, is then given by

$$
E=\frac{300}{D_{k m}}|W(\rho)|
$$

for a dipole transmitter whose strength is such that it would radiate $1 \mathrm{kw}$ over a perfectly conducting flat ground.

To illustrate the nature of the results, calculations are carried out for $E$ as a function of $D_{k m}$ over a homogeneous spherical earth of radius $6,373 \mathrm{~km}$ at frequencies of 30,150 , and $750 \mathrm{Mc}$. These curves are illustrated in figure 2, where it is indicated that the relative dielectric constant of the ground is 4 and the conductivity is $10^{-2} \mathrm{mho} / \mathrm{m}$. The flatearth formula (i. e., $\delta \rightarrow 0$ ) is designated as the zero order or Oth approximation. The results for including the first and second curvature corrections are designated as first- and second-order approximation, respectively. Finally, the field strengths obtained from the residue series are shown for comparison and designated by "Res." In this latter case many terms of the series were required, and it is reassuring to notice that curvature corrected flatearth formulas merge with the residue-series formula. The results of these calculations would lend confidence to the use of eq (40) for stratified media if the

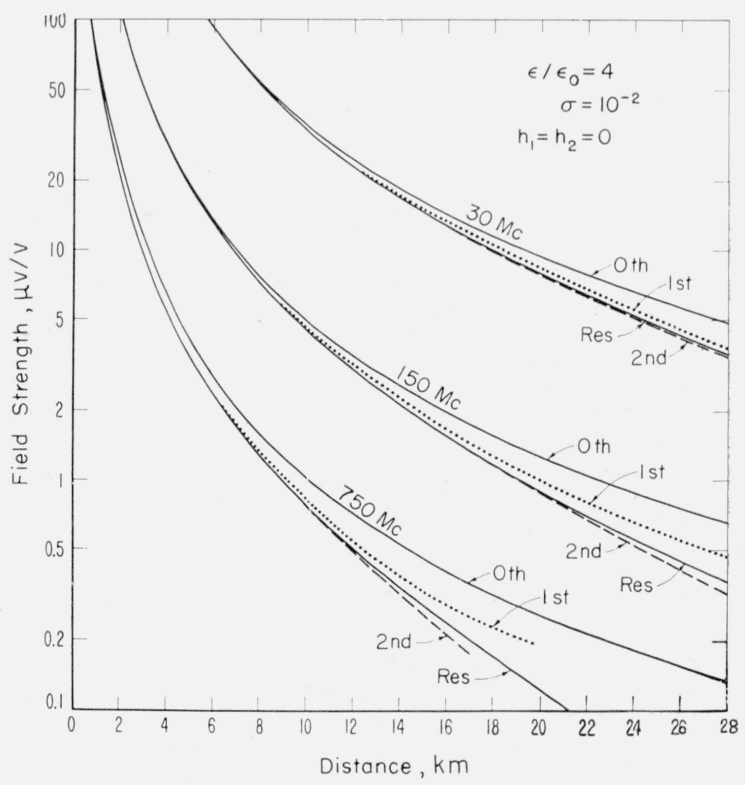

Figure 2. Field-strength versus distance curves for a transmitter that radiates 1 kilowatt.

The flat-earth and curvature corrected curves are shown along with the residue series-calculated curve.
In eqn. $(40)$, the definition of $F_{0}(\rho)$ is $F_{0}(\rho)=1-i(\pi \rho)^{\frac{1}{2}} e^{-\rho} \operatorname{erfc}\left(i \rho^{\frac{1}{2}}\right)$. 


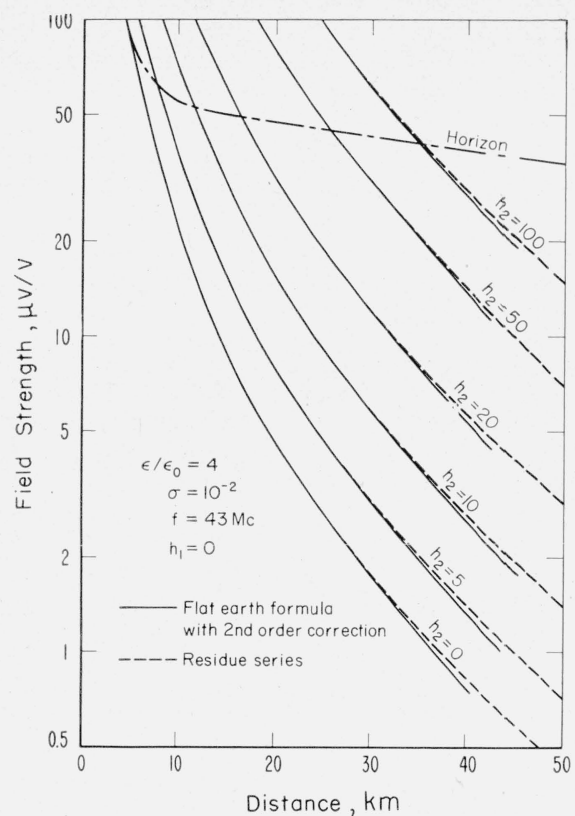

FiguRE 3. Field-strength versus distance curves for various receiving-antenna heights.

appropriate value of the numerical distance $\rho$ and curvature parameter $\delta$ are employed. As a further check on the curvature corrected formula used in conjunction with the height-gain function, calculations are carried out for various receiver heights $h_{2}$ with the transmitter height $h_{1}$ equal to zero. These results are shown in figure 3 for a frequency of $43 \mathrm{Mc}$, relative dielectric constant of 4 , conductivity of $10^{-2}$, and $h_{2}=0,5,10,20,50$, and $100 \mathrm{~m}$. The solid curves correspond to the second-order curvature corrected flat-earth formula, whereas the dashed curves are based on the cumbersome but more exact residue series [4]. As a matter of interest, the line corresponding to the optical horizon is shown. It is apparent that the agreement between the two methods of calculation is very good at moderate ranges. No doubt a third-order correction involving a term proportional to $\delta^{9}$ would improve the discrepancy at greater ranges. It is doubtful, however, if calculations of higher order correction terms are justified because the residue series becomes more convenient at larger ranges.

The computations for stratified ground are somewhat more involved, and, furthermore, even for a two-layer ground, additional parameters to be considered are conductivity $\sigma_{2}$ and dielectric constant $\epsilon_{2}$ of lower stratum, and thickness $l$ of upper stratum. As an example, the lower medium is taken to be 25 times better conducting than the upper stratum. For convenience, the dielectric constants are also taken to be in the same ratio. The second-order curvature corrected results for a frequency of $5 \mathrm{Mc}$ is shown in figure 4 , where the case $l=\infty$ corresponds to a homogeneous ground of electric constants $\sigma_{1}$ and

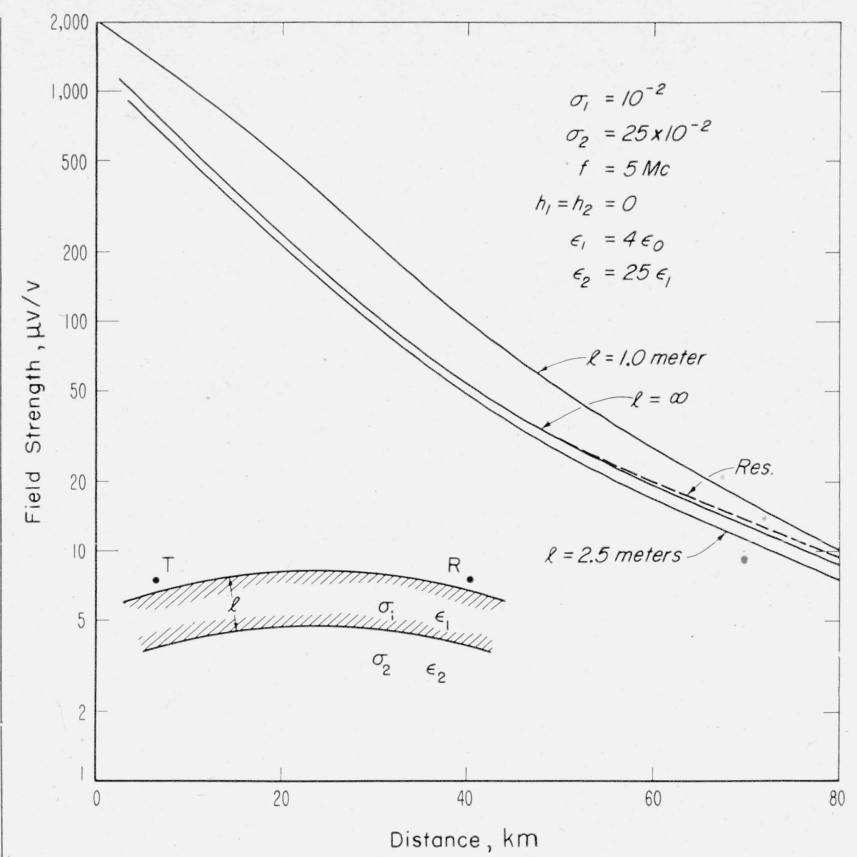

FIGURE 4. Example of the field-strength-distance curves for a two-layer stratified ground for several values of the thickness of the upper stratum.

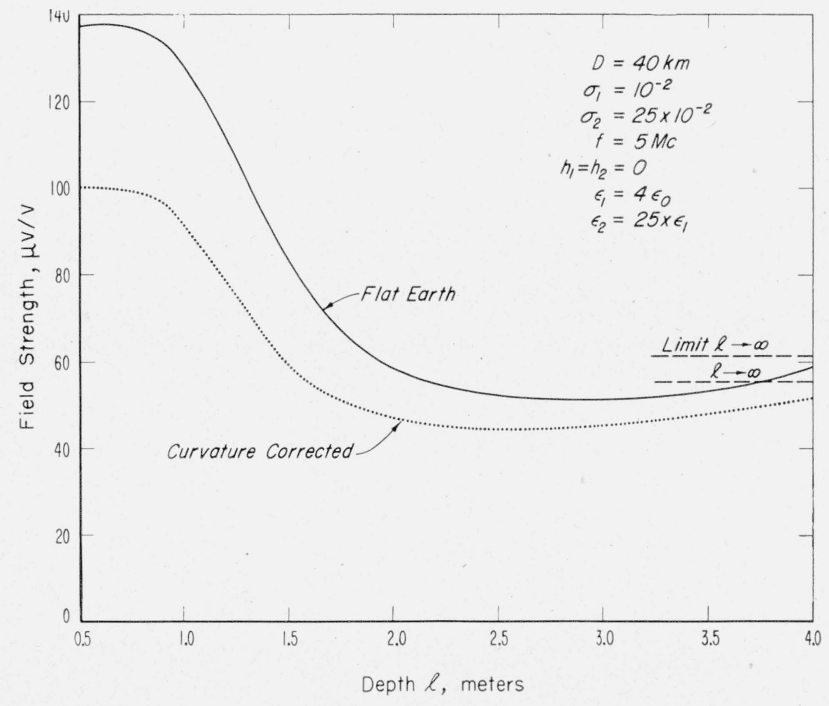

FIGURE 5. Field strength at a distance of 40 kilometers on a two-layer stratified ground as a function of the thickness of the upper stratum.

$\epsilon_{1}$. The curve marked Res. corresponds to the residue series calculation for the homogeneous earth. If the thickness of the upper stratum is greater than about $2.5 \mathrm{~m}$, the field corresponds quite closely to that expected over a homogeneous ground. To show in a little more detail, the nature of the dependence on stratification, the field at $40 \mathrm{~km}$ is shown plotted in figure 5 as a function of $l$ in meters. Both the flat-earth and the second-order curvature corrected results are included. 


\section{Concluding Remarks}

It is apparent that the theory of Watson [3], Bremmer [4], and others, for propagation of radio waves over a spherical homogeneous earth can be generalized to propagation over a concentrically stratified sphere. The derivation in this paper is very straightforward as a result of choosing an approximate boundary condition, which specifies the surface impedance at the surface of the sphere. Although the theory was developed for a homogeneous atmosphere, there is little reason to doubt that a similar analysis can be carried out for an inhomogeneous atmosphere following the development of Friedman [6]. For the case of normal refraction (absence of ducting), the results in the present paper can be employed directly by admitting the concept of the effective earth radius [4], where $a_{1}$ is replaced by a modified value $a_{1}^{e}$.

While the attention here has been mainly confined to propagation of radio waves over a stratified sphere, the results are applicable to the study of surface waves on corrugated spherical surfaces of the type that can be characterized by a surface impedance.

\section{Appendix 1. Surface Impedance of a Stratified Conducting Sphere}

The surface impedance at the surface of a sphere of radius $a_{1}$ consisting of a concentric core of radius $a_{2}$ will be derived. The propagation constants of the outer shell and the core are $\gamma_{1}$ and $\gamma_{2}$, respectively, and the intrinsic impedances are $\eta_{1}$ and $\eta_{2}$, respectively. Under the assumption that the source is a vertical antenna the field can be represented as a superposition of transverse magnetic (TM) spherical waves of order $n$. The surface impedance $Z^{n}$ for such a TM wave is defined by

$$
Z^{n}=\left[-E_{\theta}^{n} / H_{\phi}^{n}\right]_{\tau=a_{1}} .
$$

The medium between the limits $r=a_{1}$ and $a_{2}$ can now be regarded as a nonuniform transmission line [13] of length $l=a_{1}-a_{2}$. The characteristic impedance of the line looking inward is

$$
\eta_{1} \frac{\hat{I}_{n}^{\prime}\left(\gamma_{i} r\right)}{\hat{I}_{n}\left(\gamma_{1} r\right)}=M\left(\gamma_{1} r\right)
$$

where $\hat{I}_{n}$ is Schelkunoff's notation for modified spherical Bessel functions of order $n$, and the prime indicates a differentiation with respect to the argument $\gamma_{1} r$. Similarly, the characteristic impedance looking outward is

$$
-\eta_{1} \frac{\hat{K}_{n}^{\prime}\left(\gamma_{1} r\right)}{\hat{K}_{n}\left(\gamma_{2} r\right)}=N\left(\gamma_{1} r\right),
$$

where $\hat{K}_{n}$ is the modified spherical Hankel function of order $n$. The line is considered to be terminated by an impedance

$$
\left[\eta_{2} \frac{\hat{I}_{n}^{\prime}\left(\gamma_{2} r\right)}{\hat{I}_{n}\left(\gamma_{2} r\right)}\right]_{\gamma=a_{2}}=P\left(\gamma_{2} a_{2}\right) .
$$

From the theory of nonuniform transmission lines it follows that

$$
Z^{n}=M\left(a_{1}\right) \frac{1+q_{e}\left(a_{2}\right) A_{e} B_{e}}{1+q_{h}\left(a_{2}\right) A_{h} B_{h}},
$$

where $q_{e}$ and $q_{h}$ are reflection coefficients at $r=a_{2}$ for the $E_{\theta}^{n}$ and $H_{\phi}^{n}$ field components and are given by

$$
q_{e}=\frac{1 / P\left(a_{2}\right)-1 / M\left(a_{2}\right)}{1 / P\left(a_{2}\right)+1 / N\left(a_{2}\right)}
$$

and

$$
q_{h}=\frac{P\left(a_{2}\right)-M\left(a_{2}\right)}{P\left(a_{2}\right)+N\left(a_{2}\right)}
$$

The quantities $A$ and $B$ are transmission factors given by

$$
\left.\begin{array}{ll}
A_{e}=\frac{a_{1} \hat{I}_{n}^{\prime}\left(\gamma_{1} a_{2}\right)}{a_{2} \hat{I}_{n}^{\prime}\left(\gamma_{1} a_{1}\right)} & B_{e}=\frac{a_{2} \hat{K}_{n}^{\prime}\left(\gamma_{1} a_{1}\right)}{a_{1} \hat{K}_{n}^{\prime}\left(\gamma_{1} a_{2}\right)} \\
A_{h}=\frac{a_{1} \hat{I}_{n}\left(\gamma_{1} a_{2}\right)}{a_{2} \hat{I}_{n}\left(\gamma_{1} a_{1}\right)}, & B_{h}=\frac{a_{2} \hat{K}_{n}\left(\gamma_{1} a\right)}{a_{1} \hat{K}_{n}\left(\gamma_{1} a\right)}
\end{array}\right\}
$$

Under the restriction that the thickness of the shell $\left(l=a_{2}-a_{1}\right)$ is small compared to $a_{1}$, the above formulas can be greatly simplified. For example, noting that $I_{n}(x)$ satisfies the equation

$$
\frac{d^{2} \hat{I}_{n}(x)}{d x^{2}}=\left[1+\frac{n(n+1)}{z^{2}}\right] \hat{I}_{n}(x),
$$

it readily follows that the function $M(x)$ satisfies

$$
\left\{M^{2}(x)+\eta_{1} \frac{d M(x)}{d x}=\left[1+\frac{n(n+1)}{x^{2}}\right] \eta_{1}^{2}\right\} x=\gamma_{1} r .
$$

A good approximate solution is

$$
M\left(\gamma_{1} r\right) \sim_{\eta_{1}}\left[1+\frac{n(n+1)}{\left(\gamma_{1} r\right)^{2}}\right]^{1 / 2},
$$

because $d M / d x$ is small when $\left|\gamma_{1} r\right| \gg 1$. Now the values of $n$ are not known precisely, but the important ones correspond to the roots $\nu_{s}$, which are close to the value $k a_{1}$. It therefore follows that

$$
M\left(\gamma_{1} r\right) \simeq_{\eta_{1}}\left[1+\frac{k a_{1}\left(k a_{1}+1\right)}{\left(\gamma_{1} r\right)^{2}}\right]^{1 / 2} \simeq_{\eta}\left[1-\frac{\gamma_{0}^{2}}{\gamma_{1}^{2}}\right]^{1 / 2},
$$


since $k=i \gamma_{0}, k a_{1} \gg 1$ and $a_{1} / r \simeq 1$ in the range $a_{2} \leq r \leq a_{1}$. With similar reasoning it follows that

$$
N\left(\gamma_{1} r\right) \simeq \eta_{1}\left[1-\frac{\gamma_{0}^{2}}{\gamma_{1}^{2}}\right]^{1 / 2} \text { for } \quad a \leq r \leq a
$$

and

$$
P\left(\gamma_{2} a_{2}\right) \simeq \eta_{2}\left[1-\frac{\gamma_{0}^{2}}{\gamma_{2}^{2}}\right]^{1 / 2}
$$

The transmission factors are then written

$$
A_{e} \simeq A_{h} \simeq B_{e} \simeq B_{h} \simeq e^{-\left(\gamma_{1}^{2}-\gamma_{0}^{2}\right)^{1 / 2} l}
$$

with $l=a_{1}-a_{2}$. The surface impedance then takes the form

$$
Z^{n} \simeq_{\eta_{1}}\left[1-\frac{\gamma_{0}^{2}}{\gamma_{1}^{2}}\right]^{1 / 2} \frac{\frac{\gamma_{1}^{2}}{\gamma_{2}^{2}}\left[\frac{\gamma_{2}^{2}-\gamma_{0}^{2}}{\gamma_{1}^{2}-\gamma_{0}^{2}}\right]^{1 / 2}+\tanh \left[\left(\gamma_{1}^{2}-\gamma_{0}^{2}\right)^{1 / 2} l\right]}{1+\frac{\gamma_{1}^{2}}{\gamma_{2}^{2}}\left[\frac{\gamma_{2}^{2}-\gamma_{0}^{2}}{\gamma_{1}^{2}-\gamma_{0}^{2}}\right]^{1 / 2} \tanh \left[\left(\gamma_{1}^{2}-\gamma_{0}^{2}\right)^{1 / 2} l\right]} .
$$

It is to be noted that $Z^{n}$ is precisely the ratio $Z$ of the tangential electric and magnetic fields for a vertically polarized wave at grazing incidence on a two-layer stratified ground [5]. It, therefore, seems justified to employ the approximate boundary eq (7) at least for application to propagation of radio waves over the surface of a stratified ground with the normal earth curvature.

\section{Appendix 2. Alternative Approach to the Height-Gain Function}

It is possible to study the effect of raising the receiver and/or the transmitter by starting with a more accurate representation for the height-gain function:

$$
f\left(p, h_{i}\right)=\frac{h_{\nu}^{(2)}(k r)}{h_{\nu}^{(2)}(k a)},
$$

where

$$
\nu=k a+(k a)^{1 / 3}\left[-\frac{p}{2 \delta^{2}}\right]
$$

with $r=h_{i}+a ; f\left(p, h_{i}\right)$ is now expanded in a MacLaurin series as follows:

$$
\begin{aligned}
& f(p, h)=1+k h_{i}\left[\frac{\frac{d}{d x} h_{\nu}^{(2)}(x)}{h_{\nu}^{(2)}(x)}\right]_{x=\kappa a} \\
&+\frac{k^{2} h_{i}^{2}}{2}\left[\frac{\frac{d^{2}}{d x^{2}} h_{\nu}^{(2)}(x)}{h_{\nu}^{(2)}(x)}\right]_{x=k a}+\cdots
\end{aligned}
$$

Now employing the relation

$$
\frac{1}{x} \frac{d}{d x} \log x h_{\nu}^{(2)}(x) \simeq \frac{i \Delta}{x}
$$

and the differential equation

$$
\frac{d^{2}}{d x^{2}}+\frac{2}{x} \frac{d}{d x}+\left[1-\frac{\nu(\nu+1)}{x^{2}}\right] h_{\nu}^{(2)}(x)=0,
$$

it follows that

$$
f\left(p, h_{i}\right) \simeq 1+\left(i \Delta k h_{i}+\frac{h_{i}}{a}\right)-\frac{i \Delta k^{2} h_{i}^{2}}{k a}+\frac{k^{2} h_{i}^{2} \Delta}{2} .
$$

It then follows that a more representative heightgain function $G^{\prime}(\rho)$ in place of the function $G$ is given by the operator

$$
G^{\prime}(\rho) \simeq 1+i k h_{i} \Delta-i \frac{k^{2} h_{i}^{2} \Delta}{k a}+\frac{k^{2} h_{i}^{2} \Delta}{2} \frac{\partial}{\partial \rho}+\cdots
$$

The field $E(\rho, h)$ is then related to the field $E(\rho, 0)$ on the ground by $E(\rho, h)=G^{\prime}(\rho) E(\rho, 0)$. The third term of the expansion is negligible when $k h<(k a)^{1 / 3}$ because $|\Delta|$ is somewhat less than unity and the fourth term is negligible when

$$
k h_{i}^{2} / D \ll 1 .
$$

The function $G^{\prime}(\rho)$ to this approximation is then identical to the height-gain function $G$ obtained earlier.

\section{References}

[1] J. R. Wait, Radiation from a vertical dipole over a (plane) stratified ground, Trans. Inst. Radio Engrs. $\mathbf{A P}-1,9$ (1953); AP- $\mathbf{2}, 144$ (1954).

[2] J. R. Wait, Theory of electromagnetic surface waves over geological conductors, Geofis. pura e appl. 28, 47 (1954).

[3] G. N. Watson, The diffraction of radio waves by the earth, Proc. Roy. Soc. (London) [A] 95, 546 (1919).

[4] H. Bremmer, Terrestrial radio waves (Elsevier Publishing Co., 1949).

[5] J. R. Wait, Propagation of radio waves over a stratified ground, Geophysics 18, 416 (1953).

[6] B. F. Freidman, Propagation in a non-homogeneous atmosphere, in Theory of electromagnetic waves (Interscience Publishers, New York, N. Y., 1951).

[7] R. S. Elliott, Spherical surface wave antennas. Paper presented at URSI Symposium on Electromagnetic Theory at Ann Arbor, Mich. (to be published in Trans. Inst. Radio Engrs.).

[8] H. J. Josephs, Heaviside's electric circuit theory (Methuen \& Co. Ltd., London, 1946).

[9] G. A. Campbell and R. M. Foster, Fourier integrals for practical application, p. 92, No. 811 (1948).

[10] K. A. Norton, The calculation of the ground wave field intensity, Proc. Inst. Radio Engrs. 29, 723 (1941).

[11] A. N. Sommerfeld, Uber die Ausbreitung der Wellen in der drachtlosen Telegraphie, Ann. Physik 81, 1135 (1926).

[12] G. A. Hufford, An integral equation approach to the problem of wave propagation over an irregular surface, Quart. Appl. Math. 9, 391 (1952).

[13] S. A. Schelkunoff, Electromagnetic waves (Van Nostrand Co., Inc., New York, N. Y., 1943).

Boulder, August 30, 1955. 